\title{
Application of molecular targeted therapy for refractory metastatic thyroid cancers
}

\author{
Timothy Allen ${ }^{1 *}$ and Lee Meller ${ }^{2}$ \\ ${ }^{1}$ Global Allied Pharmaceutical, Center for Excellence in Research \& Development, USA \\ ${ }^{2}$ inVentiv Health, USA
}

\begin{abstract}
Thyroid cancer is one of the leading causes of death worldwide. There have been several risks factors linked with thyroid cancer such as genetics, exposure to radiation, body weight and insulin resistance. Poorly differentiated and recurrent metastatic thyroid cancer cell carcinoma remains a therapeutic challenge because of its strong resistance to both chemotherapy and radiation therapy. Immunotherapy might play a role in the treatment of patients with metastatic thyroid cancer. In this paper, we discuss the potential causes of thyroid cancer, pathophysiology, and currently available molecular targeted therapy for refractory metastatic thyroid cancers and common side effects of treatment.
\end{abstract}

\begin{abstract}
Abbreviations: ATC: Anaplastic thyroid carcinoma, CEA: Carcinoembryonic antigen, CSC: Cancer stemness cell, CTL: Cytotoxic T-lymphocyte, DTC: Differentiated thyroid carcinoma, EGFR: Epidermal growth factor receptor, FTC: Follicular thyroid carcinoma, MTC: Medullary thyroid carcinoma, MAPK: Mitogen-activated protein kinase, PI3K: Phosphatidylinositol-3-kinase, PTC: Papillary thyroid carcinoma, RTK: Receptor tyrosine kinase, SEER: The Surveillance, Epidemiology, and End Results, SJS: Stevens-Johnson syndrome, TEN: Toxic epidermal necrolysis, VEGFR: Vascular endothelial growth factor receptor 2
\end{abstract}

\section{Introduction/Epidemiology}

Thyroid cancer arises in the follicular or para-follicular cells of the thyroid glands. Thyroid cancer is one of the leading causes of death worldwide. The Surveillance, Epidemiology, and End Results (SEER) estimated 62,980 (47,790 in females, and 15,190 in males) new cases and 1,890 ( 1,060 females and 830 males) cases of death in the United States in 2014. Based on the prevalence of thyroid cancer, it was estimated that there were 566,708 people alive with thyroid cancer in the United States in 2011. Thyroid cancer represents $3.8 \%$ of all the cancer cases in the United States. The five year survival rate of thyroid cancer was $97.8 \%$ from the year 2004 to 2010 in the United States [1]. The overall worldwide estimated age-standardized incidence and mortality rates were $2.1 \%$ and $0.5 \%$ respectively [2]. It includes different type of histopathologic and genetic characteristics. The male to female ratio of the incidence of thyroid cancer is 3:1 [3].

\section{Etiology/Predisposing factors}

Thyroid cancer is due to the abnormal growth of cells in the thyroid gland secondary to switching on or off of the tumor suppressor or promoter genes.

Risk factors for thyroid cancer include:

Gender and age: Thyroid cancer occurs more often in females as compared to males. Thyroid cancer typically occurs between 40 to 50 years in females and 60-70 years in males.
A diet low in iodine: Follicular thyroid cancer and papillary thyroid cancer are more common in people, with low dietary iodine intake.

Exposure of radiation: Radiation (nuclear weapons, radioactive plants, radiation therapy.) is a proven risk factor the thyroid cancer.

Other risk factors: Thyroid nodules, certain inherited genetic syndromes [4], TSH levels, body weight and insulin resistance [5].

\section{Types of thyroid cancer}

Thyroid cancer has four subtypes which include [6]:

- Follicular thyroid carcinomas (FTC): Invasion into vascular structures is common and therefore metastasis to other tissues

- Papillary thyroid carcinomas (PTC): Papillary thyroid carcinoma is the most common type of thyroid cancer. The growth rate of PTC is very slow and often develop in only one lobe of the thyroid. This cancer often spreads to the lymph nodes in the neck

- Medullary thyroid carcinomas (MTC): Medullary thyroid carcinoma develops from the thyroid gland $\mathrm{C}$ cells, which produces calcitonin hormone. This hormone controls the level of calcium in the blood. This cancer accounts for only $4 \%$ of all the thyroid cancers.

- Anaplastic thyroid carcinomas (ATC): which accounts for $2 \%$ of all the thyroid cancers. ATC is an undifferentiated carcinoma, which is very aggressive and difficult to treat.

Correspondence to: Timothy Allen, MD, Ph.D, Global Allied Pharmaceutical, Center for Excellence in Research \& Development, 160 Vista Oak Dr. Longwood, FL 32779, USA, Tel: 1-321-945-4283, E-mail: timothy.allen@gapsos.com

Key words: thyroid cancer, targeted therapy, cancer treatment, immunotherapy, tumour targeting

Received: May 13, 2016; Accepted: June 13, 2016; Published: June 17, 2016 
Anaplastic thyroid carcinoma occurs due to the changes in CTNNB1 oncogenes and TP53 tumor suppressor gene. Follicular thyroid carcinoma occurs due to the changes in RAS oncogenes. Papillary thyroid carcinoma occurs due to the changes in BRAF tumor suppressor genes and RET/PTC oncogenes. Medullary thyroid carcinoma occurs due to the inherited mutation of RET.

Most thyroid cancers present as asymptomatic thyroid nodules identified on physical exam or as incidental findings during diagnostic imaging. The most common signs and symptoms of thyroid cancer are: a lump in the neck, increasing hoarseness, difficulty in swallowing and breathing, swollen lymph nodes, and pain in the neck and throat.

\section{Pathophysiology and molecular basis}

There are several pathways involved in the molecular pathophysiology of thyroid cancer. The RAS mutation, BRAF mutation, MAP kinase signaling pathway, PI3K/Akt signaling pathway, PAX8$\operatorname{PPAR} \gamma$ rearrangement, $\mathrm{p} 53$ inactivation and epigenetic regulation are the basic pathways in thyroid cancer.

RAS mutations: The most important pathway in thyroid cancer is the RAS pathway, which is vital for the growth of papillary thyroid carcinomas (PTC) and also plays a role in follicular thyroid carcinomas (FTC) [7]. There are three types of RAS genes: N-RAS, K-RAS, and $\mathrm{H}-\mathrm{RAS}$, and these genes produce $21-\mathrm{kDa}$ proteins [8]. The activation of RAS oncogene due to the point mutation also affects the GTPase domain (codon 61) in exon 2 or the GTP-binding domain (codons 12 or 13) in exon 1, which his keeps the protein in the activated form [9]. Some studies demonstrated that RAS mutations, occurs specifically at codon 61 of N-RAS $[10,11]$.

BRAF mutation and MAP kinase signaling pathway: BRAF mutation plays a key role in the activation of mitogen-activated protein kinase (MAPK) signaling pathway in cancer [12]. There are three types of RAF kinases: A-Raf, B-Raf (BRAF), and C-Raf. Among these RAF kinases, BRAF is the most effective activator of MAPK pathway [13]. This is the outcome of the addition of 18 nucleotides at nucleotide T1799 [14-16]. Thus, the BRAF mutation is a molecular marker for thyroid cancer. The BRAF pathway, which appears to be essential for the growth of (PTC) also plays an additional role in (FTC) [17].

PI3K/Akt signaling pathway: The genetic modification of proteins inside the phosphatidylinositol-3-kinase (PI3K)/AKT signaling pathway have been reported in sporadic thyroid carcinomas, (FTC) and (ATC) [18]. The phosphatidylinositol-3 kinase (PI3K)/Akt signaling pathway (PI3K pathway) also plays an essential role in the survival, cell growth and proliferation and formation of tumors, similar to that of the MAP kinase pathway $[19,20]$. The class I of PI3Ks is made up of heterodimers of a regulatory subunit, mainly p85 and p110 catalytic subunits. The $\alpha$-type (PIK3CA) and $\beta$-type (PIK3CB) p110 subunits are found in all tissues, while other p110 subunits are found in the limited tissues. There are three main types of Akts: Akt-1, Akt-2, and Akt-3 [21]. These Akt isoforms are present in abundance in thyroid cancer cells. The amplification of PIK3CA is found mainly in ATC and FTC [22,23].

PAX8-PPAR $\gamma$ rearrangement: The PAX8-PPAR $\gamma$ rearrangement is a chromosomal translocation $\mathrm{t}(2: 3)(\mathrm{q} 13 ; \mathrm{p} 25)$, which promotes the growth of thyroid cancers [24]. Paired-box gene 8 (PAX-8) encodes a transcription factor, which is necessary for the growth of thyroid follicular cell lineage and thyroid-specific gene expression in thyroid cancer [25]. The PAX8-PPAR $\gamma$ rearrangement hints to the in-frame synthesis of exon 7, 8, or 9 of PAX8 on 2q13 through exon 1 of PPAR $\gamma$ on $3 p 25$ [26]. The PAX8-PPAR $\gamma$ rearrangement is also involved in the growth of thyroid cancer through RAS mutation [27].

p53 inactivation: The tumor suppressor gene (TP53) is located on chromosome 17 and is frequent in ATC and PDTC. It may be responsible for the loss of differentiation in these tumors [7]. Alterations in the p53 tumor suppressor gene by inactivating point mutations, usually involving exons $5-8$, or by deletion result in additional mutations, progressive genome destabilization and propagation of malignant clones. It represents the most common genetic abnormality in cancer, which occurs typically as late tumorigenic events [28-30].

Epigenetic regulation: The epigenetic changes, such as the altered DNA methylation in the CpG islands of the gene promoters, are vital in the usual thyrocyte function, such as the TSH receptor and the sodiumiodide symporter [31,32]. The elementary structural part of chromatin contains 147 bp of DNA that enfolds about an octamer of four essential histone proteins such as $\mathrm{H} 2 \mathrm{~A}, \mathrm{H} 2 \mathrm{~B}, \mathrm{H} 3$, and $\mathrm{H} 4$ [33]. The different histone modification comprises ubiquitination, phosphorylation, acetylation, and methylation that acts with DNA promoter methylation to regulate gene silencing [34] (Figure 1).

\section{Immunotherapy overview}

Targeted therapy might offer therapeutic options for patients with medullary thyroid cancers, advanced cases that don't respond to radioactive iodine therapy and anaplastic thyroid carcinomas. These therapies are not curative, but may slow the progression of the tumor. The cost effectiveness of targeted agents in advanced thyroid cancer has not been established, because the drugs are so new. Targeted therapies are generally better tolerated than traditional chemotherapy with the most common side effects of biological therapies are Diarrhea fatigue, rash, cardiac dysfunction, thrombosis and hypertension Clinical studies are needed to identify patients most likely to benefit from these therapies.

Current immunotherapy options for thyroid cancer are discussed in following categories: kinase inhibitors, monoclonal antibodies, mTOR inhibitors, proteasome inhibitors, vaccine therapy and cancer cell stemness inhibitors.

\section{Kinase inhibitors}

\section{FDA approved kinase inhibitors}

Sorafenib: It is a kinase inhibitor that blocks the enzyme RAF kinase, a critical component of the RAF/MEK/ERK signaling pathway that controls cell division and proliferation. In addition, Sorafenib inhibits the VEGFR-2/PDGFR-beta signaling cascade, thereby blocking tumor angiogenesis. Sorafenib is indicated for the treatment of metastatic or recurrent, progressive, differentiated thyroid carcinoma (DTC), refractory to ablative radioactive iodine treatment. It is contraindicated in combination with taxane and platinum chemotherapy [35].

Its in vitro binding to human plasma protein is $99.5 \%$. Sorafenib is metabolized by CYP3A4 and steady state plasma is reached within 7 days, with a half-life of 25-48 hours. Sorafenib should not be given to patients with cardiac ischemia. It should be discontinued in case of bleeding. Women of reproductive potential should be advised not to get pregnant as Sorafenib can harm the fetus. Sorafenib should be discontinued for dermatologic toxicities, such as toxic epidermal necrolysis (TEN) and Stevens-Johnson syndrome (SJS). The most common adverse effects are hemorrhage, hypertension, and gastrointestinal. 


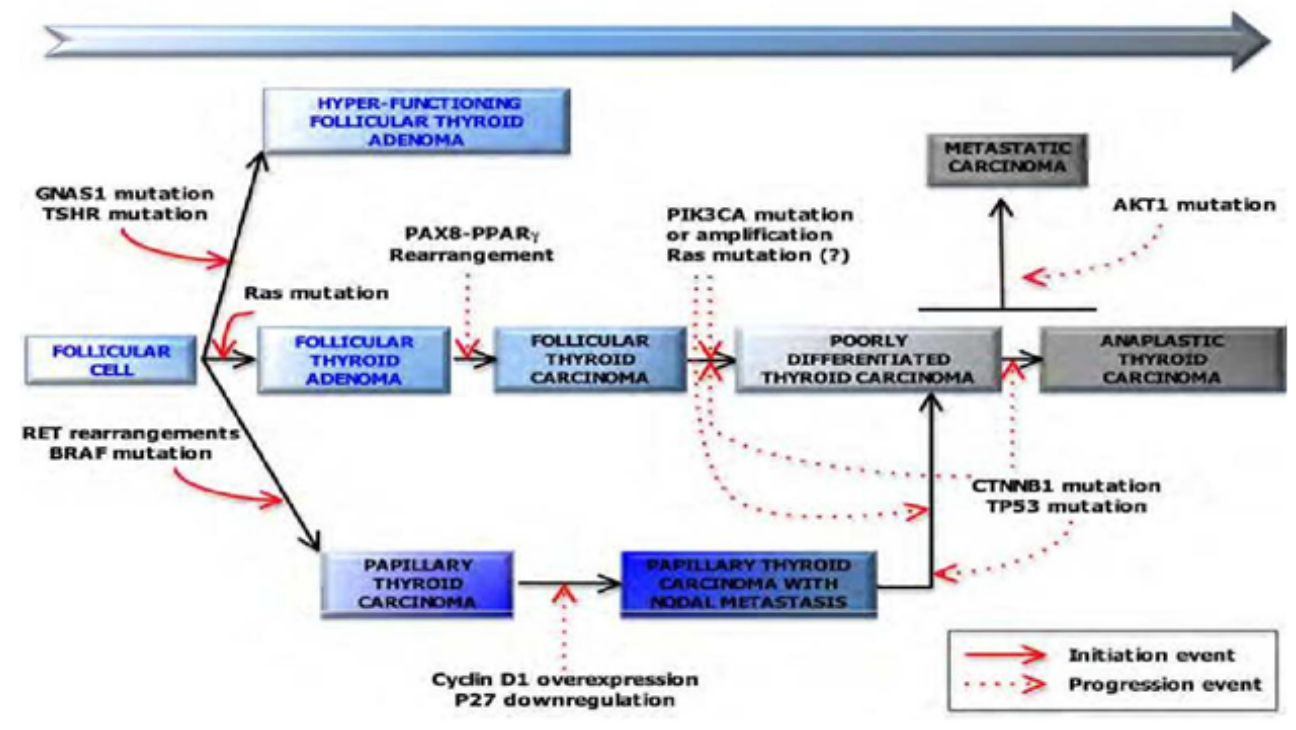

Figure 1. Multi-step mechanism of thyroid cancer [7].

Vandetanib: Vandetanib selectively inhibits the tyrosine kinase activity of vascular endothelial growth factor receptor 2 (VEGFR2), thereby blocking VEGF-stimulated endothelial cell proliferation, migration and reducing tumor vessel permeability. This agent also blocks the tyrosine kinase activity of epidermal growth factor receptor (EGFR), a receptor tyrosine kinase that mediates tumor cell proliferation, migration and angiogenesis. It is used for the treatment of symptomatic or progressive medullary thyroid cancer in patients with unresectable, locally advanced, or metastatic disease [36].

The action of vandetanib remains unaffected by the food. Vandetanib binds to a1-acid-glycoprotein and exhibits $90 \%$ in vitro protein binding. It is metabolized through the CYP3A4 with a half-life is 21 days.

The most common adverse effects are gastrointestinal. Vandetanib should not be given to patients with long QT syndrome, hypocalcemia, hypomagnesaemia, and hypokalemia. Vandetanib should be discontinued for dermatologic toxicities. Women of reproductive potential should be advised not to get pregnant it can harm the fetus. Vandetanib should be discontinued in patients with ischemic cerebrovascular diseases.

Cabozantinib: A small molecule receptor tyrosine kinase (RTK) inhibitor with potential antineoplastic activity. Cabozantinib strongly binds to and inhibits several RTKs, which are often overexpressed in a variety of cancer cell types, including hepatocyte growth factor receptor (MET), RET (rearranged during transfection), vascular endothelial growth factor receptor types 1 (VEGFR-1), 2 (VEGFR-2), and 3 (VEGFR-3), mast/stem cell growth factor (KIT), FMS-like tyrosine kinase 3 (FLT-3), TIE-2 (TEK tyrosine kinase, endothelial), tropomyosin-related kinase $\mathrm{B}$ (TRKB) and AXL. This may result in an inhibition of both tumor growth and angiogenesis, and eventually lead to tumor regression. It is used for the treatment of progressive, metastatic medullary thyroid cancer patients [37].

Its binding to human plasma protein is $99.7 \%$ and steady state is attained within 15 days. It is metabolized through CYP3A4. The most common adverse effects are gastrointestinal. Cabozantinib should not be administered in patients with severe hemorrhage. Cabozantinib should be discontinued in patients with cerebral infarction, myocardial infarction, or arterial thromboembolus

Lenvatinib: A synthetic, orally available inhibitor of vascular endothelial growth factor receptor 2 (VEGFR2, also known as KDR/ FLK-1) tyrosine kinase with potential antineoplastic activity. Lenvatinib mesylate blocks VEGFR2 activation by VEGF, resulting in inhibition of the VEGF receptor signal transduction pathway, decreased vascular endothelial cell migration and proliferation, and vascular endothelial cell apoptosis [38].

Lenvima (lenvatinib) is used to treat patients with progressive, differentiated thyroid cancer (DTC) whose disease progressed despite receiving radioactive iodine therapy (radioactive iodine refractory disease).

There are some kinase inhibitors that are not currently approved by FDA for thyroid cancer. However, many kinase inhibitors are under clinical trials in phase I, II, and III as in Table 1.

\section{Monoclonal antibody (mAb)}

There are no mAbs that are currently approved by FDA for thyroid cancer. However, the $\mathrm{mAb}$ under clinical trials in phase I- III is given in Table 2.

Bevacizumab: A recombinant humanized monoclonal antibody directed against the vascular endothelial growth factor (VEGF), a proangiogenic cytokine. Bevacizumab binds to VEGF and inhibits VEGF receptor binding, thereby preventing the growth and maintenance of tumor blood vessels.

\section{mTOR inhibitors}

Few mTOR inhibitors are under clinical trials in phase I-III are listed in Table 3.

Everolimus: It is a derivative of natural macrocyclic lactone sirolimus and has anti-angiogenic and immunosuppressant properties. It binds to FKBP-12 to generate an immunosuppressive complex and inhibits the activation of mTOR. Everolimus is an orally bioavailable inhibitor of the mTOR pathway. The effects of Everolimus in cancer 
Table 1. Non-FDA approved kinase inhibitor Drugs [39-49].

\begin{tabular}{|l|l|l|l|l|}
\hline Drug & Clinical trial identifier number & Phase & Study design \\
\hline Dabrafenib & NCT01723202 & Phase II & Randomized, Open Label, Efficacy Study \\
\hline Vemurafenib & NCT02145143 & Phase II & Safety/Efficacy Study, Open label \\
\hline Selumetinib & NCT01843062 & Phase III & Safety study, Double blind \\
\hline Ponatinib & NCT01838642 & Phase II & Non-Randomized, Open Label, Efficacy Study \\
\hline Sunitinib & NCT01396408 & Phase II & Non-Randomized, Open Label, Safety/Efficacy Study VEGFR2, PDGFRb \\
\hline E7080 & NCT01728623 & Phase II & Open Label, Safety/Efficacy Study \\
\hline Nintedanib & NCT01788982 & Safety/Efficacy Study, Double blind \\
\hline Pazopanib & NCT01813136 & Phase II & Randomized, Open Label, Efficacy Study \\
\hline Crizotinib & NCT02034981 & Phase II & Open Label, Efficacy Study \\
\hline Trametinib & NCT02152995 & Phase II & Open Label, Efficacy Study \\
\hline Ceritinib & NCT02289144 & Phase II & Open Label, Safety/Efficacy Study \\
\hline
\end{tabular}

Table 2. Non-FDA Approved mAB drug [50].

\begin{tabular}{|l|l|l|l|l|}
\hline mAb & Clinical trial identifier number & Phase & Study design \\
\hline Bevacizumab & NCT00804830 & Phase II & Non-Randomized, Open label, Efficacy Study & VEGF \\
\hline
\end{tabular}

Table 3: Non-FDA approved mTOR Drugs [51,52].

\begin{tabular}{|l|l|l|l|}
\hline Drug & Clinical trial identifier number & Phase & Study design \\
\hline Everolimus & NCT01270321 & Phase II & Randomized, Open Label, Efficacy Study \\
\hline MLN0128 & NCT02244463 & Phase II & Open Label, Efficacy Study \\
\hline
\end{tabular}

Table 4. Non-FDA approved vaccines [53].

\begin{tabular}{|l|l|l|l|l|l|l}
\hline Drugs & Clinical trial identifier number & Phase & Study design \\
\hline GI-6207 & NCT01856920 & Phase I & Randomized, Open Label, Safety/Efficacy Study & CTL \\
\hline
\end{tabular}

Table 5. Non-FDA approved drugs [55].

\begin{tabular}{|c|c|c|c|c|}
\hline Drugs & Clinical trial identifier number & Phase & Study design & Target \\
\hline BBI608 & NCT01325441 & Phase I/II & Non-Randomized, Open Label, Safety/Efficacy Study & $\mathrm{CSC}$ \\
\hline
\end{tabular}

cells include reduced growth and proliferation as well as inhibition of protein translation and VEGF production.

MLN0128: An inhibitor of raptor-mTOR (TOR complex 1 or TORC1) and rictor-mTOR (TOR complex 2 or TORC2) with potential antineoplastic activity. TORC1/2 inhibitor MLN0128 binds to and inhibits both TORC1 and TORC2 complexes of mTOR, which may result in tumor cell apoptosis and a decrease in tumor cell proliferation. TORC1 and 2 are upregulated in some tumors and play an important role in the PI3K/Akt/mTOR signaling pathway, which is frequently dysregulated in human cancers.

\section{Vaccines}

There are no vaccines that are currently approved by FDA for thyroid cancer. However, the vaccie that is under clinical trials in phase I- III is given in Table 4.

GI-6207: A whole, heat-killed, recombinant Saccharomyces cerevisiae yeast-based vaccine genetically altered to express the carcinoembryonic antigen (CEA) peptide $610 \mathrm{D}$ with potential immunostimulating and antineoplastic activities. Upon administration, recombinant Saccharomyces cerevisia-CEA(610D) vaccine GI-6207 may stimulate a host cytotoxic T-lymphocyte (CTL) response against CEA-expressing tumor cells, which may result in tumor cell lysis. CEA, a tumor associated antigen, is overexpressed on a wide variety of human cancer cells including colorectal, gastric, lung, breast and pancreatic cancer cells. CEA 610D encodes for 9 amino acids (605-613), in which aspartate is substituted for asparagine at position 610 (610D) in order to strengthen the induction of the CTL response against CEAexpressing tumor cells.

\section{Cancer cell stemness inhibitors}

The discovery of therapeutic approaches that counteract relapse and metastasis is, therefore, extremely important for advancing cancer medicine. Hypermalignant cancer cells, termed cancer stem cells or stemness-high cancer cells, have been isolated from patients with a variety of tumor types and found to be highly malignant, tumorigenic, and resistant to chemotherapies, A cancer stemness inhibitor effectively blocks cancer relapse and metastasis in xenografted human cancers, suggest targeting cancer stemness as a novel approach to develop the next generation of cancer therapeutics to suppress cancer relapse and metastasis [54].

BBI608: It is a cancer cell stemness inhibitor with potential antineoplastic activity. Even though the exact target has yet to be fully elucidated, BBI608 appears to target and inhibit multiple pathways involved in cancer cell stemness. This may ultimately inhibit cancer stemness cell (CSC) growth, as well as heterogeneous cancer cell growth. CSCs, self-replicating cells that are able to differentiate into heterogeneous cancer cells, appear to be responsible for the malignant growth, recurrence and resistance to conventional chemotherapies (Table 5).

\section{Conclusion}

The recent discoveries of the pathogenesis of thyroid cancer are very 
important for the growth of novel and capable therapies. Several genetic modifications that are recognized so far, concentrate on the PI3K/ AKT, RTK/RAS, and BRAF/MAPK signaling pathways, contributes to a powerful foundation for the growth of novel therapeutic, prognostic, and gene-based strategies. The treatment of thyroid cancer is altered significantly as a result of the development of kinase inhibitors. It is not yet resolved whether the various combinations of targeted therapies with radiotherapy or chemotherapy will improve the response rates of advanced and difficult to treat thyroid cancer.

\section{References}

1. SEER Stat Fact Sheets: Thyroid Cancer. SEER.[http://seer.cancer.gov/statfacts/html/ thyro.html].

2. International Agency for Research on Cancer.[http://globocan.iarc.fr/Pages/fact sheets_population.aspx].

3. Davies L, Welch HG (2014) Current thyroid cancer trends in the United States. JAMA Otolaryngol Head Neck Surg 140: 317-322.[Crossref]

4. Thyroid cancer. National Cancer Institute.[http://www.cancer.gov/cancertopics/types/ thyroid].

5. (2013) Cancer facts and figures.[http://www.cancer.org/acs/groups/content/@ epidemiologysurveilance/documents/document/acspc-036845.pdf].

6. Pellegriti G, Frasca F, Regalbuto C, Squatrito S, Vigneri R (2013) Worldwide increasing incidence of thyroid cancer: update on epidemiology and risk factors. $J$ Cancer Epidemiol 2013: 965212.[Crossref]

7. Viglietto G and De Marco C. Molecular Biology of Thyroid Cancer. Contemporary Aspects of Endocrinology. 2011, 189-234, Available from: http://cdn.intechopen.com/ pdfs-wm/24139.pdf.

8. Barbacid M (1978) RAS genes. Annual Review of Biochemistry 56.

9. Meinkoth JL (2004) Biology of Ras in thyroid cells. Cancer Treat Res 122: 131-148. [Crossref]

10. Esapa CT, Johnson SJ, Kendall-Taylor P, Lennard TW, Harris PE (1999) Prevalence of Ras mutations in thyroid neoplasia. Clin Endocrinol (Oxf) 50: 529-535.[Crossref]

11. Motoi N, Sakamoto A, Yamochi T, Horiuchi H, Motoi T, et al. (2000) Role of ras mutation in the progression of thyroid carcinoma of follicular epithelial origin. Pathol Res Pract 196: 1-7.[Crossref]

12. Mercer KE, Pritchard CA (2003) Raf proteins and cancer: B-Raf is identified as a mutational target. Biochim Biophys Acta 1653: 25-40.[Crossref]

13. Garnett MJ, Marais R (2004) Guilty as charged: B-RAF is a human oncogene. Cancer Cell 6: 313-319.[Crossref]

14. Moretti S, Macchiarulo A, De Falco V, Avenia N, Barbi F, et al. (2006) Biochemical and molecular characterization of the novel BRAF(V599Ins) mutation detected in a classic papillary thyroid carcinoma. Oncogene 25: 4235-4240.[Crossref]

15. Oler G, Ebina KN, Michaluart P Jr, Kimura ET, Cerutti J (2005) Investigation of BRAF mutation in a series of papillary thyroid carcinoma and matched-lymph node metastasis reveals a new mutation in metastasis. Clin Endocrinol (Oxf) 62: 509-511.[Crossref]

16. Hou P, Liu D, Xing M (2007) Functional characterization of the T1799-1801del and A1799-1816ins BRAF mutations in papillary thyroid cancer. Cell Cycle 6: 377-379. [Crossref]

17. Ciampi R, Knauf JA, Kerler R, Gandhi M, Zhu Z, et al. (2005) Oncogenic AKAP9BRAF fusion is a novel mechanism of MAPK pathway activation in thyroid cancer. $J$ Clin Invest 115: 94-101. [Crossref]

18. Ringel MD, Hayre N, Saito J, Saunier B, Schuppert F, et al. (2001) Overexpression and overactivation of Akt in thyroid carcinoma. Cancer Res 61: 6105-6111.[Crossref]

19. Mian C, Barollo S, Pennelli G, Pavan N, Rugge M, et al. (2008) Molecular characteristics in papillary thyroid cancers (PTCs) with no 131I uptake. Clin Endocrinol (Oxf) 68: 108-116.[Crossref]

20. Vivanco I, Sawyers CL (2002) The phosphatidylinositol 3-Kinase AKT pathway in human cancer. Nat Rev Cancer 2: 489-501.[Crossref]

21. Fresno Vara JA, Casado E, de Castro J, Cejas P, Belda-Iniesta C, et al. (2004) PI3K/Akt signalling pathway and cancer. Cancer Treat Rev 30: 193-204.[Crossref]

22. García-Rostán G, Costa AM, Pereira-Castro I, Salvatore G, Hernandez R, et al. (2005)
Mutation of the PIK3CA gene in anaplastic thyroid cancer. Cancer Res 65: 1019910207.[Crossref]

23. Hou P, Liu D, Shan Y, Hu S, Studeman K, et al. (2007) Genetic alterations and their relationship in the phosphatidylinositol 3-kinase/Akt pathway in thyroid cancer. Clin Cancer Res 13: 1161-1170.[Crossref]

24. Kroll TG, Sarraf P, Pecciarini L, Chen CJ, Mueller E, et al. (2000) PAX8-PPARgammal fusion oncogene in human thyroid carcinoma [corrected]. Science 289: 1357-1360. [Crossref]

25. Desvergne B, Wahli W (1999) Peroxisome proliferator-activated receptors: nuclear control of metabolism. Endocr Rev 20: 649-688.[Crossref]

26. Tallini G (2002) Molecular pathobiology of thyroid neoplasms. EndocrPathol 13: 271288.[Crossref]

27. Ying H, Suzuki H, Zhao LI, Willingham MC, Meltzer P, et al. (2003) Mutant thyroid hormone receptor $\beta$ represses the expression and transcriptional activity of peroxisome proliferator-activated receptor? during thyroid carcinogenesis. Cancer Res 63: 52745280.[Crossref]

28. Segev DL, Umbricht C, Zeiger MA (2003) Molecular pathogenesis of thyroid cancer Surg Oncol 12: 69-90.[Crossref]

29. Donghi R, Longoni A, Pilotti S, Michieli P, Della Porta G, et al. (1993) Gene p53 mutations are restricted to poorly differentiated and undifferentiated carcinomas of the thyroid gland. J Clin Invest 91: 1753-1760.[Crossref]

30. La Perle KM, Jhiang SM, Capen CC (2000) Loss of p53 promotes anaplasia and local invasion in ret/PTC1-induced thyroid carcinomas. Am J Pathol 157: 671-677.[Crossref]

31. Xing M (2007) Gene methylation in thyroid tumorigenesis. Endocrinology 148: 948 953.[Crossref]

32. Akagi T, Luong QT, Gui D, Said J, Selektar J, et al. (2008) Induction of sodium iodide symporter gene and molecular characterisation of HNF3ß/FoxA2, TTF-1 and C/EBPB in thyroid carcinoma cells. Br J Cancer 99: 781-788.

33. Kouzarides T (2007) Chromatin modifications and their function. Cell 128: 693-705. [Crossref]

34. Ting AH, McGarvey KM, Baylin SB (2006) The cancer epigenome--components and functional correlates. Genes Dev 20: 3215-3231.[Crossref]

35. FDA approved label NEXAVAR (sorafenib) Manufactured by Bayer HealthCare Pharmaceuticals Inc. last updated on 2013.[http://www.accessdata.fda.gov/drugsatfda docs/label/2013/021923s016lbl.pdf].

36. FDA approved label Vandetanib (vandetanib) Manufactured by AstraZeneca Pharmaceuticals LP. Last updated on 2011.[ http://www.accessdata.fda.gov/drugsatfda docs/label/2011/022405s000lbl.pdf].

37. FDA approved label COMETRIQTM (cabozantinib) Manufactured by Exelixis, Inc. last updated on 2012.[ http://www.accessdata.fda.gov/drugsatfda_docs/ label/2012/203756lbl.pdf].

38. http://www.accessdata.fda.gov/drugsatfda_docs/label/2015/206947s000lbl.pdf.

39. National Comprehensive Cancer Network. Shah M, Ohio State University Comprehensive Cancer Center. Dabrafenib with or without Trametinib in Treating Patients with Recurrent Thyroid Cancer. In: ClinicalTrials.gov [Internet]. Bethesda (MD): National Library of Medicine (US). 2014DEC27.

40. Memorial Sloan-Kettering Cancer Center, Memorial Sloan-Kettering Cancer Center Enhancing Radioiodine (RAI) Incorporation into BRAF Mutant, RAI-Refractory Thyroid Cancers with the BRAF Inhibitor Vemurafenib: A Pilot Study. In: ClinicalTrials gov [Internet]. Bethesda (MD): National Library of Medicine (US). 2014DEC27.

41. AstraZeneca; AstraZeneca. Study Comparing Complete Remission after Treatment With Selumetinib/Placebo in Patient With Differentiated Thyroid Cancer (ASTRA) In: ClinicalTrials.gov [Internet]. Bethesda (MD): National Library of Medicine (US). 2014 DEC27.

42. National Cancer Institute (NCI). Ponatinib for Advanced Medullary Thyroid Cancer. In: ClinicalTrials.gov [Internet]. Bethesda (MD): National Library of Medicine (US) 2015 FEB27.

43. NCIC Clinical Trials Group; NCIC Clinical Trials Group. A Phase II Study of Sunitinib or Temsirolimus in Patients With Advanced Rare Tumours. In: ClinicalTrials.gov [Internet]. Bethesda (MD): National Library of Medicine (US). 2015April18

44. Eisai Co., Ltd; Eisai Inc. (Eisai Co., Ltd.). A Study of E7080 in Subjects With Advanced Thyroid Cancer. In: ClinicalTrials.gov [Internet]. Bethesda (MD): National Library of Medicine (US). 2015April18. 
45. European Organisation for Research and Treatment of Cancer - EORTC. Nintedanib (BIBF1120) in Thyroid Cancer. In: ClinicalTrials.gov [Internet]. Bethesda (MD): National Library of Medicine (US). 2015 July 6.

46. Centre Leon Berard; Centre Leon Berard. Phase II Study of the Optimal Scheme of Administration of Pazopanib in Thyroid Carcinoma. In: ClinicalTrials.gov [Internet]. Bethesda (MD): National Library of Medicine (US). 2015April18.

47. UNICANCER; UNICANCER. Phase 2 Study Assessing Efficacy and Safety of Crizotinib in Patients Harboring an Alteration on ALK, MET or ROS1 (AcSé). In: ClinicalTrials.gov [Internet]. Bethesda (MD): National Library of Medicine (US) 2015April18.

48. National Cancer Institute (NCI); National Cancer Institute (NCI). Trametinib in Increasing Tumoral Iodine Incorporation in Patients With Recurrent or Metastatic Thyroid Cancer. In: ClinicalTrials.gov [Internet]. Bethesda (MD): National Library of Medicine (US). 2015April18.

49. University of Texas Southwestern Medical Center; University of Texas Southwestern Medical Center. Ceritinib (LDK378) in Mutation and Oncogene Directed Therapy in Metastatic or Locally Advanced Anaplastic/Undifferentiated Thyroid Cancer. In: ClinicalTrials.gov [Internet]. Bethesda (MD): National Library of Medicine (US). 2015 April18.
50. Region Skane; Region Skane. Avastin and Doxorubicin Postoperatively for Patients with Anaplastic Thyroid Cancer. In: ClinicalTrials.gov [Internet]. Bethesda (MD): National Library of Medicine (US). 2014DEC27.

51. Emory University; Taofeek K. Owonikoko, Emory University. A Trial of Pasireotide and Everolimus in Adult Patients with Radioiodine-Refractory Differentiated and Medullary Thyroid Cancer. In: ClinicalTrials.gov [Internet]. Bethesda (MD): National Library of Medicine (US). 2014DEC27.

52. Dana-Farber Cancer Institute; JochenLorch, MD, Dana-Farber Cancer Institute. A Phase II Study of MLN0128 in Metastatic Anaplastic Thyroid Cancer. In: ClinicalTrials.gov [Internet]. Bethesda (MD): National Library of Medicine (US). 2015April18.

53. GlobeImmune; GlobeImmune. A Phase 2 Study of GI-6207 in Patients With Recurrent Medullary Thyroid Cancer. In: ClinicalTrials.gov [Internet]. Bethesda (MD): National Library of Medicine (US). 2015April18.

54. Li Y, Rogoff HA, Keates S, Gao Y, Murikipudi S, et al. (2015) Suppression of cancer relapse and metastasis by inhibiting cancer stemness. Proc Natl Acad Sci U S A 112 1839-1844.[Crossref]

55. Boston Biomedical, Inc; Boston Biomedical, Inc. A Study of BBI608 Administered With Paclitaxel in Adult Patients With Advanced Malignancies. In: ClinicalTrials.gov [Internet]. Bethesda (MD): National Library of Medicine (US). 2015April18.

Copyright: @2016 Allen T. This is an open-access article distributed under the terms of the Creative Commons Attribution License, which permits unrestricted use, distribution, and reproduction in any medium, provided the original author and source are credited. 